\title{
DO JETS PRECESS... OR EVEN MOVE AT ALL?
}

\author{
Chris Nixon ${ }^{1,2,3}$ AND ANDREw King ${ }^{2}$ \\ ${ }^{1}$ JILA, University of Colorado \& NIST, Boulder, CO 80309-0440, USA; chris.nixon@jila.colorado.edu \\ 2 Department of Physics and Astronomy, University of Leicester, University Road, LE1 7RH Leicester, UK \\ Received 2012 September 25; accepted 2013 January 14; published 2013 February 13
}

\begin{abstract}
Observations of accreting black holes often provoke suggestions that their jets precess. The precession is usually supposed to result from a combination of the Lense-Thirring effect and accretion disk viscosity. We show that this is unlikely for any type of black hole system, as the disk generally has too little angular momentum compared with a spinning hole to cause any significant movement of the jet direction across the sky on short timescales. Uncorrelated accretion events, as in the chaotic accretion picture of active galactic nuclei (AGNs), change AGN jet directions only on timescales $\gtrsim 10^{7} \mathrm{yr}$. In this picture AGN jet directions are stable on shorter timescales, but uncorrelated with any structure of the host galaxy, as observed. We argue that observations of black hole jets precessing on timescales short compared to the accretion time would be a strong indication that the accretion disk, and not the standard Blandford-Znajek mechanism, is responsible for driving the jet. This would be particularly convincing in a tidal disruption event. We suggest that additional disk physics is needed to explain any jet precession on timescales short compared with the accretion time. Possibilities include the radiation warping instability, or disk tearing.
\end{abstract}

Key words: accretion, accretion disks - black hole physics - galaxies: active - galaxies: evolution - galaxies: jets

\section{INTRODUCTION}

Jets appear in all accreting systems, from protostars (e.g., Davis et al. 1994) to active galactic nuclei (AGNs; e.g., Nagar \& Wilson 1999; Kinney et al. 2000). In all cases the terminal speed of the jet is $\gtrsim$ the escape speed from the surface of the accreting object. Studies of protostellar jets usually assume that the ultimate power source is the accretion energy of the gas disk forming the star, mediated by strong magnetic fields (e.g., Price et al. 2012 and references therein). To tap the maximum accretion energy, a jet produced in this way must come from the innermost part of the disk near the stellar surface, and so naturally gives a terminal velocity of the order of the escape speed. For black holes, there is debate as to whether the jet driver is again the disk accretion energy (e.g., Blandford \& Payne 1982; Livio et al. 1999) or instead the black hole spin (Blandford \& Znajek 1977).

Observations of jets from AGN often encourage suggestions that the jets precess (e.g., Falceta-Gonçalves et al. 2010; Kharb et al. 2010; Gong et al. 2011; Martí-Vidal et al. 2011). For the two suggested types of black hole jet driving, this requires precession either of the disk plane close to the central accretor (where the jet is launched), or instead, of the black hole spin axis. In this Letter, we consider these processes and show that precessing jets are not easy to obtain via any of the mechanisms usually invoked. The reasons are simply: (1) the angular momentum of any single realistic accretion event is always smaller than the angular momentum of the hole; and (2) the inner disk settles rapidly into a steady shape. This is aligned to the spin if $\alpha>H / R$, and a steady warp if $\alpha<H / R$. Here $\alpha$ is the Shakura-Sunyaev viscosity parameter and $H / R$ is the disk angular semithickness, and the two cases correspond to diffusive and wave-like warp propagation, respectively.

\section{LENSE-THIRRING EFFECT IN DISKS WITH $\alpha>H / R$}

We briefly describe the evolution of a misaligned disk around a spinning black hole in the regime where warps propagate

\footnotetext{
3 Einstein Fellow.
}

diffusively-i.e., $\alpha>H / R$ (Papaloizou \& Pringle 1983). We discuss the wave-like case $(\alpha<H / R)$ in Section 3.

The diffusive case is considered at length in the literature (e.g., Bardeen \& Petterson 1975; Pringle 1992; Scheuer \& Feiler 1996; Lodato \& Pringle 2006; Nixon \& King 2012). The Lense-Thirring effect of a spinning black hole makes tilted disk orbits precess around its angular momentum vector at a frequency $\Omega_{\mathrm{LT}}=a\left(R / R_{\mathrm{g}}\right)^{-3} \Omega_{\mathrm{K}}\left(R_{\mathrm{g}}\right)$ (where $a$ is the Kerr spin parameter, $R_{\mathrm{g}}=G M / c^{2}$ is the black hole's gravitational radius, and $\Omega_{\mathrm{K}}\left(R_{\mathrm{g}}\right)$ is the Kepler frequency at disk radius $\left.R_{\mathrm{g}}\right)$, which decreases strongly with radius (Thirring 1918; Lense \& Thirring 1918). This differential precession is communicated through the disk by its viscosity, which acts to co- or counter-align the disk with the plane of the hole. The inner parts of the disk quickly settle in the equatorial plane of the black hole and the outer parts remain misaligned, with the two parts joined by a warped region. This is the Bardeen-Petterson effect (Bardeen \& Petterson 1975; but note that the equations of that paper do not conserve angular momentum; see Papaloizou \& Pringle 1983). If an external torque (e.g., from a misaligned binary companion) maintains the tilt at the outer edge of the disk the warp can remain stationary, but otherwise the warp propagates outward until the entire disk lies in the equatorial plane. The hole-disk system thus ends up aligned (or counter-aligned) along its original total angular momentum (the vector sum of the original spin and disk angular momenta; King et al. 2005). We note that so far all calculations of the Bardeen-Petterson effect have used Shakura \& Sunyaev (1973) $\alpha$ disks; a demonstration of the effect for disks explicitly driven by the magnetorotational instability (MRI) has not yet been attempted.

The Bardeen-Petterson evolution assumes that the disk viscosity is strong enough to communicate the differential precession efficiently through the disk. Recently, Nixon \& King (2012) and Nixon et al. (2012a) have shown that for realistic parameters this often does not hold. Instead, the disk is torn into many distinct planes that precess almost independently of each other (Nixon et al. 2012a). If the disk inclination to the black hole spin is high enough, this generates significantly counterrotating disk orbits and these lead to rapid accretion (cf. Nixon 
et al. 2012b). These results markedly alter the picture of how black holes accrete, and may allow for strong precession of the inner disk plane. We return to this possibility in Section 4, but for the moment consider the usual Bardeen-Petterson evolution.

To discuss possible jet precessions we let $\boldsymbol{J}_{\mathrm{d}}, \boldsymbol{J}_{\mathrm{h}}$, and $\boldsymbol{J}_{\mathrm{t}}=\boldsymbol{J}_{\mathrm{d}}+\boldsymbol{J}_{\mathrm{h}}$ be the disk, hole, and total angular momentum vectors respectively, with magnitudes $J_{\mathrm{d}}, J_{\mathrm{h}}$, and $J_{\mathrm{t}}$. During the alignment process, $\boldsymbol{J}_{\mathrm{h}}$ precesses around $\boldsymbol{J}_{\mathrm{t}}$ with an initial amplitude $\theta_{\mathrm{i}}$ defined by

$$
\cos \theta_{\mathrm{i}}=\frac{\boldsymbol{J}_{\mathrm{h}} \cdot \boldsymbol{J}_{\mathrm{t}}}{J_{\mathrm{h}} J_{\mathrm{t}}} .
$$

This angle is small (i.e., $\boldsymbol{J}_{\mathrm{t}}$ and $\boldsymbol{J}_{\mathrm{h}}$ are in a similar direction) either when the disk is oriented in a similar direction to the hole, or when $J_{\mathrm{d}} \ll J_{\mathrm{h}}$ (and so $\boldsymbol{J}_{\mathrm{h}} \simeq J_{\mathrm{t}}$ ).

It is clear that if $J_{\mathrm{d}} \ll J_{\mathrm{h}}$ alignment cannot move the hole spin vector very far. The inner disk must quickly become anchored to the spin plane of the hole (e.g., King et al. 2005), so alignment cannot move the inner disk very far either. So if $J_{\mathrm{d}} \ll J_{\mathrm{h}}$ the Lense-Thirring effect cannot drive a precessing jet.

Thus if we have the usual Bardeen-Petterson evolution, precessions are confined at best to cases where $J_{\mathrm{d}} \gtrsim J_{\mathrm{h}}$. However, this still does not generate repeated jet precession. The initial amplitude of the precession can be large, since $J_{\mathrm{t}} \gg J_{\mathrm{h}}$. But the alignment and precession timescales for the disk are similar (Scheuer \& Feiler 1996): after only one precession time the hole is significantly aligned with the disk. This is shown explicitly in Lodato \& Pringle (2006), who get at most a single precession of the jet (see their Figures 6 and 11) with significant amplitude.

We conclude that in a tilted disk propagating warps in the diffusive regime $(\alpha>H / R)$, the Lense-Thirring effect alone cannot drive repeated jet precession, unless the disk is torn into many distinct planes (Nixon et al. 2012a).

\subsection{Do Jets Move?}

We have argued above that sustained Lense-Thirring precessions are inhibited by the dynamics of the disk-hole system. We now ask how much angular momentum can be transferred from an accretion event on to a black hole. In particular, can this change its direction significantly? We derive a simple expression for $J_{\mathrm{d}} / J_{\mathrm{h}}$ and use it to consider realistic parameters for various astrophysical systems.

The disk angular momentum is

$$
J_{\mathrm{d}} \sim M_{\mathrm{d}}\left(G M R_{\mathrm{d}}\right)^{1 / 2}=M_{\mathrm{d}} R_{\mathrm{d}} V_{\mathrm{K}}\left(R_{\mathrm{d}}\right),
$$

where $M_{\mathrm{d}}$ is the disk mass, $M$ is the black hole mass, $R_{\mathrm{d}}$ a characteristic radius for the disk, $V_{\mathrm{K}}$ the Keplerian velocity, and $G$ is the gravitational constant.

The spin angular momentum of a black hole with dimensionless spin parameter $a$ is (Kumar \& Pringle 1985)

$$
J_{\mathrm{h}}=\frac{G M^{2} a}{c},
$$

where $c$ is the speed of light. Combining (2) and (3) gives us

$$
\frac{J_{\mathrm{d}}}{J_{\mathrm{h}}}=\frac{1}{a} \frac{M_{\mathrm{d}}}{M} \frac{R_{\mathrm{d}}}{R_{\mathrm{g}}} \frac{V_{\mathrm{K}}}{c},
$$

or equivalently

$$
\frac{J_{\mathrm{d}}}{J_{\mathrm{h}}}=\frac{1}{a} \frac{M_{\mathrm{d}}}{M}\left(\frac{R_{\mathrm{d}}}{R_{\mathrm{g}}}\right)^{1 / 2}
$$

where $R_{\mathrm{g}}=G M / c^{2} \sim 10^{13} M_{8} \mathrm{~cm}$ is the gravitational radius (with $M_{8}=M / 10^{8} M_{\odot}$ ). It is clear that this ratio can take very different values for various astrophysical systems, as we now consider.

\subsubsection{Tidal Disruption Events}

In a tidal disruption event, a star on a near-parabolic orbit around a supermassive black hole fills its tidal lobe near pericenter and is torn apart. This condition implies a pericenter separation $p$ given by

$$
p \simeq\left(\frac{M}{M_{*}}\right)^{1 / 3} R_{*},
$$

where the star has mass and radius $M_{*}, R_{*}$. Since $R_{\mathrm{d}}<p$ and $M_{\mathrm{d}}<M_{*}$, we find

$$
\frac{J_{\mathrm{d}}}{J_{\mathrm{h}}}<\frac{1}{a}\left(\frac{M_{*}}{M}\right)^{5 / 6}\left(\frac{R_{*}}{R_{\mathrm{g}}}\right)^{1 / 2}
$$

Even in the most favorable case of a giant star $\left(R \sim 10^{13} \mathrm{~cm}\right)$, (7) implies a tiny ratio

$$
\frac{J_{\mathrm{d}}}{J_{\mathrm{h}}} \lesssim 3 \times 10^{-7} M_{8}^{-1 / 2} .
$$

This makes it obvious that any observational evidence for the movement (let alone precession) of a jet in a tidal disruption event is incompatible with jet driving by the hole spin, as is central to the standard axisymmetric Blandford-Znajek mechanism. If instead it is assumed that the jet is driven by the inner accretion disk, this must involve physics more complex than a standard thin disk warped by the Lense-Thirring effect. Tidal disruption events may produce geometrically thick disks and therefore could propagate warps as waves (see Section 3), but this requires $\alpha$ to be unusually small (cf. King et al. 2007).

\subsubsection{Black Hole Binaries}

This case appears slightly more promising than a tidal disruption as the black hole and the donor star have comparable masses $M_{1}, M_{2}$, with $0.1 \lesssim M_{2} / M_{1} \lesssim 10$. However, at any one instant only a small fraction of the donor star feeds the black hole and thus again we have $M_{\mathrm{d}} \ll M$. As favorable parameters we take $R_{\mathrm{g}} \approx 3 \times 10^{6} \mathrm{~cm}$ (i.e., a $10 M_{\odot}$ black hole), and a large disk radius $R_{\mathrm{d}} \lesssim 10^{13} \mathrm{~cm}$. The largest realistic disk mass is $M_{\mathrm{d}} \lesssim 10^{-5} M_{\odot}$ (e.g., Equation (5.51) of Frank et al. 2002 with viscosity parameter $\alpha=0.1$ and an accretion rate $\dot{M}=10^{19} \mathrm{~g} \mathrm{~s}^{-1}$ corresponding to the Eddington limit for a $10 M_{\odot}$ black hole). This gives

$$
\frac{J_{\mathrm{d}}}{J_{\mathrm{h}}}=\frac{1}{a} \frac{M_{\mathrm{d}}}{M_{1}}\left(\frac{R_{\mathrm{d}}}{R_{\mathrm{g}}}\right)^{1 / 2} \lesssim \frac{10^{-3}}{a} .
$$

Thus the disk has far too little instantaneous angular momentum to cause the hole spin axis to move on a directly observable timescale. We again conclude that jet movement would imply that the jet is not driven by the black hole spin, or by the alignment of a standard thin disk warped by the Lense-Thirring effect. We note that if the disk is geometrically thick it could propagate warps as waves (see Section 3), but this requires $\alpha$ to be unusually small (cf. King et al. 2007). 


\subsubsection{Active Galactic Nuclei}

This case has been considered by King et al. (2008). The main constraint on $J_{\mathrm{d}}$ is the fact that disks that are too large tend to fragment into stars under self-gravity. King et al. (2008) show that a maximal disk of this type has $J_{\mathrm{d}} / J_{\mathrm{h}} \lesssim$ few $\times 10^{-2} a^{-1}$ and has an instantaneous mass $\sim 10^{-3} M$, where $M$ is the SMBH mass. Thus a mass $\sim 0.01 \mathrm{a} M$ must pass through this kind of disk, with constant orientation, to move the direction of a centrally produced jet by $\sim 0.1$ radian. This would take at least $10^{-2} a$ Salpeter times, i.e., $\lesssim 4 \times 10^{5} a$ yr, even with continuous accretion at the Eddington rate, and typically $\gtrsim 10^{7} a$ yr if accretion is slower and slightly intermittent. If the orientation of successive accretion disk events changes randomly, as envisaged in the chaotic accretion picture of AGN (King \& Pringle 2006, 2007), the spin direction would perform a random walk and so deviate less from its original direction.

We again conclude that detectable jet precession is unlikely in AGN. In the chaotic accretion picture, jets generally move very little for timescales $\lesssim$ a few $\times 10^{6}$ yr. However, a sequence of significant but random accretion events can move AGN jets across the sky on longer timescales $\left(\gtrsim 10^{7} \mathrm{yr}\right)$. These conclusions agree with the facts that jets with relatively stable or closely correlated directions are seen (e.g., Kharb et al. 2006), but jet directions do not correlate at all with any features of the host galaxy (Kinney et al. 2000).

\section{LENSE-THIRRING EFFECT IN DISKS WITH $\alpha<H / R$}

We have argued above that Lense-Thirring precession in standard thin disks cannot be responsible for repeated precessions of jets. However, it is unlikely that the innermost regions of black hole accretion disks remain thin. In this section, we discuss the possibility of precession in disks with $H / R>\alpha$. We again find that repeated precession of the jet is generally unlikely, but this time not impossible.

In Section 2 we assumed $\alpha>H / R$, so that warps propagate diffusively. But if $\alpha<H / R$, warps instead propagate efficiently as waves with near-sonic velocities, and are not locally damped by viscosity. It is therefore possible that the transmission of such waves in the inner disk region could produce a precession. However, this requires quite specific initial conditions-i.e., that the accreting material be arranged into a radially narrow ring, and $\alpha$ must be small. If instead the radial extent of the disk is large, the wave induced by the Lense-Thirring effect propagates outward, and either never returns (on timescales of interest) or significantly damps before returning (the wave has to reach the outer disk edge before reflecting back inward). Lubow et al. (2002) give an example where the disk has $R_{\text {out }} / R_{\text {in }}=90$ with $H / R=0.1$ and $\alpha=0.05$. In this case the inner disk effectively settles into a steady shape while the wave slowly propagates to the outer disk. As Lubow et al. (2002) remark (last paragraph of their Section 4), "the steady-state shape of the disk close to the hole is essentially established." The disk quickly sets up a shape in which the internal disk torques balance the Lense-Thirring precession torque. Thus for any precession to occur and move the jet, the inner regions must wait for the outward propagating wave to reach a boundary and reflect back inward. The reflection timescale is $\sim 2 R_{\text {out }} / c_{\mathrm{s}}$ (e.g., Nixon \& Pringle 2010), where $R_{\text {out }}$ is the distance the wave must travel and $c_{\mathrm{s}} / 2$ is the wave speed (Papaloizou \& Lin 1995).

This reasoning is not inconsistent with the simulations of Fragile et al. (2007), which suggest repeated precession of a tilted disk around a black hole. Here the authors do not assume an $\alpha$ viscosity, but instead simulate the MRI in an inclined thick disk $(H / R \sim 0.2)$. As is known to happen in such cases (e.g., King et al. 2007), this implies an effective viscosity parameter $(\alpha \approx 0.01)$ rather lower than implied by observations $(\alpha \approx 0.1-0.3)$. Figure 13 of Fragile et al. (2007) shows the value of alpha in their computation, ranging from $\alpha \approx 0.5$ near the innermost stable circular orbit (ISCO) to $\alpha \approx 2 \times 10^{-3}$ in the center of their disk $\left(R=25 R_{\mathrm{g}}\right)$ to $\alpha \approx$ a few $\times 10^{-4}$ in the outer parts ( $R \approx 50 R_{\mathrm{g}}$ ). Away from the ISCO these values are far from those inferred from observations or those predicted by shearing box simulations (e.g., Simon et al. 2012). This may well be because the simulation run time is not long enough to allow the MRI to develop fully; for example, the run time is $\sim 10$ orbits at $R=25 R_{\mathrm{g}}$, and only $\sim 3$ orbits at $50 R_{\mathrm{g}}$. We note that the disk precession (Figure 16 of Fragile et al. 2007) is averaged over the disk region $20 R_{\mathrm{g}}<R<50 R_{\mathrm{g}}$. We also note that the timescale on which the disk is expected to reach a steady (not precessing) shape is $\sim 1 /(\alpha \Omega)$ (see Equation (4) of Lubow et al. 2002). This timescale is much longer than the runtime of the simulations showing precession. Longer runs are needed to check whether for realistic viscosities and disk sizes the repeated precession observed in Fragile et al. (2007) remains, rather than damping away after only a few orbits of the disk.

A thick $(H / R \gtrsim \alpha) \operatorname{small}\left(R \ll c_{\mathrm{s}} t_{\text {damp }}\right)$ disk can in principle precess. If one can arrange a disk like this to make a sharp transition (on a scale length less than the warp wavelength) to a thin disk outside it, the wave could see this as a hard boundary and efficiently reflect back inward. The dynamics of such a setup is largely unexplored, but since the thick region is fed by the thin region, a minimum condition is that the tilt in the thin region must be maintained. This requires extra physics, as we advocate below.

The disk geometry needed for repeated precession in the wave-like regime is feasible for a tidal disruption event, where the gas circularizes very close to the accreting black hole, and the instantaneous accretion rate can be super-Eddington. However, this is again problematic: for a thick disk with $H / R \sim 0.1$ and $\alpha \sim 0.1$, the inner disk $\left(R<10 R_{\mathrm{g}}\right)$ aligns after at most a few precessions (Equation (35) of Bate et al. 2000).

\section{DISCUSSION}

We have argued that the physics of standard warped disks (diffusive or wave-like) strongly suggests that the Lense-Thirring effect alone is not a promising mechanism for explaining jet precessions, except possibly in rather rare cases (see Section 3). The essential reason for this is that the accretion disk generally has total angular momentum small compared with that of the spinning black hole, strongly restricting the motion of any jet across the sky. Two alternative mechanisms, so far largely unexplored, may offer more promising ways of moving jets.

First, Pringle (1996) shows that an accretion disk can be unstable to warping driven by irradiation from a central source. If there is initially a small tilt in the disk, this can grow to provide a substantial global tilt in the disk with the angle between inner and outer parts differing by up to $\Delta \theta \sim \pi$. The inner regions of the disk precess with a quasi-periodic change in inclination (Pringle 1997). This mechanism uses the angular momentum induced by anisotropic scattering of the central accretion luminosity, so could potentially be more powerful than the Lense-Thirring effect.

A second possibility for large precessions of the disk plane close to the black hole is that for large disk tilts it may break into distinct planes, with only tenuous viscous communication 
between them. This happens when the Lense-Thirring torque is strong enough to overcome the viscous torques holding the disk together (Nixon \& King 2012; Nixon et al. 2012a). Nixon et al. (2012a) show that rapid precessions can occur here. We shall explore these ideas in future papers.

Finally, we note that the interaction of the jet with superEddington winds coming from the disk can also generate precession of the jet as suggested for SS433 (Begelman et al. 2006). Here the jet collides with a precessing gas mass and is deflected (and slowed). The jet precession here is purely a consequence of the deflection.

We thank Phil Armitage for useful discussions. Support for this work was provided by NASA through the Einstein Fellowship Program, grant PF2-130098. Research in theoretical astrophysics at Leicester is supported by an STFC Rolling Grant.

\section{REFERENCES}

Bardeen, J. M., \& Petterson, J. A. 1975, ApJL, 195, L65

Bate, M. R., Bonnell, I. A., Clarke, C. J., et al. 2000, MNRAS, 317, 773

Begelman, M. C., King, A. R., \& Pringle, J. E. 2006, MNRAS, 370, 399

Blandford, R. D., \& Payne, D. G. 1982, MNRAS, 199, 883

Blandford, R. D., \& Znajek, R. L. 1977, MNRAS, 179, 433

Davis, C. J., Mundt, R., \& Eisloeffel, J. 1994, ApJL, 437, L55

Falceta-Gonçalves, D., Caproni, A., Abraham, Z., Teixeira, D. M., \& de Gouveia Dal Pino, E. M. 2010, ApJL, 713, L74

Fragile, P. C., Blaes, O. M., Anninos, P., \& Salmonson, J. D. 2007, ApJ, 668,417
Frank, J., King, A., \& Raine, D. J. 2002, Accretion Power in Astrophysics (3rd ed.; Cambridge: Cambridge Univ. Press)

Gong, B. P., Li, Y. P., \& Zhang, H. C. 2011, ApJL, 734, L32

Kharb, P., Hota, A., Croston, J. H., et al. 2010, ApJ, 723, 580

Kharb, P., O’Dea, C. P., Baum, S. A., Colbert, E. J. M., \& Xu, C. 2006, ApJ, 652,177

King, A. R., Lubow, S. H., Ogilvie, G. I., \& Pringle, J. E. 2005, MNRAS, 363,49

King, A. R., \& Pringle, J. E. 2006, MNRAS, 373, L90

King, A. R., \& Pringle, J. E. 2007, MNRAS, 377, L25

King, A. R., Pringle, J. E., \& Hofmann, J. A. 2008, MNRAS, 385, 1621

King, A. R., Pringle, J. E., \& Livio, M. 2007, MNRAS, 376, 1740

Kinney, A. L., Schmitt, H. R., Clarke, C. J., et al. 2000, ApJ, 537, 152

Kumar, S., \& Pringle, J. E. 1985, MNRAS, 213, 435

Lense, J., \& Thirring, H. 1918, PhyZ, 19, 156

Livio, M., Ogilvie, G. I., \& Pringle, J. E. 1999, ApJ, 512, 100

Lodato, G., \& Pringle, J. E. 2006, MNRAS, 368, 1196

Lubow, S. H., Ogilvie, G. I., \& Pringle, J. E. 2002, MNRAS, 337, 706

Martí-Vidal, I., Marcaide, J. M., Alberdi, A., et al. 2011, A\&A, 533, A111

Nagar, N. M., \& Wilson, A. S. 1999, ApJ, 516, 97

Nixon, C., King, A., Price, D., \& Frank, J. 2012a, ApJL, 757, L24

Nixon, C. J., \& King, A. R. 2012, MNRAS, 421, 1201

Nixon, C. J., King, A. R., \& Price, D. J. 2012b, MNRAS, 422, 2547

Nixon, C. J., \& Pringle, J. E. 2010, MNRAS, 403, 1887

Papaloizou, J. C. B., \& Lin, D. N. C. 1995, ApJ, 438, 841

Papaloizou, J. C. B., \& Pringle, J. E. 1983, MNRAS, 202, 1181

Price, D. J., Tricco, T. S., \& Bate, M. R. 2012, MNRAS, 423, L45

Pringle, J. E. 1992, MNRAS, 258, 811

Pringle, J. E. 1996, MNRAS, 281, 357

Pringle, J. E. 1997, MNRAS, 292, 136

Scheuer, P. A. G., \& Feiler, R. 1996, MNRAS, 282, 291

Shakura, N. I., \& Sunyaev, R. A. 1973, A\&A, 24, 337

Simon, J. B., Beckwith, K., \& Armitage, P. J. 2012, MNRAS, 422, 2685

Thirring, H. 1918, PhyZ, 19, 33 